PÓSTER 3

\title{
Aplicación de Evaluación Clínica y Atención Temprana de la Po- tencialidad Cerebromotriz Innata en los Recién Nacidos Vivos con Factores de Riesgo Neonatal del Servicio de Neonatología del Hospital General San Vicente de Paúl
}

\begin{tabular}{ll}
\hline AUTOR: & Marco Antonio Montesdeoca Bastidas \\
INSTITUCCIÓN: & Hospital General San Vicente De Paúl \\
PAIS: & Ibarra, Provincia de Imbabura-Ecuador
\end{tabular}

\section{RESUMEN}

Introducción: En el Hospital General San Vicente de Paúl de la ciudad de Ibarra, se observó un porcentaje considerable en nacimientos de recién nacidos vivos con factores de riesgo neonatal, por lo que emerge la iniciativa en la evaluación Clínica y Atención en Intervención Temprana de la Potencialidad Cerebromotriz Innata. Materiales y Métodos: Se realizó un estudio Longitudinal Panel Mixto prospectivo, por recolectar los datos a través del tiempo en períodos, ejecutando deducciones respecto al cambio, sus determinantes y consecuencias. La muestra la constituyeron 340 neonatos que fueron evaluados en la Motricidad Bucofacial, 118 Neonatos en el Potencial Motor Innato y 83 Neonatos Prematuros en seguimiento de Atención e Intervención Temprana. Al inicio se evaluó determinando los trastornos Transitorios o Patológicos conforme a la Escala de Guy Tardieu, para luego proceder a la aplicación de técnicas en Intervención Temprana. Resultados: En un $87 \%$ de (" $n "=83$ ) se detectó la presencia de Trastornos Cerebromotrices Transitorios, con Atención e Intervención Temprana desde la hospitalización neonatal y el seguimiento en consulta externa se habilitaron y mejoraron la Potencialidad Cerebromotriz llegando a etapas de desarrollo neuromotor correspondiente a la normalidad, y en un 13\% se encontró alteraciones patológicas como Parálisis Cerebral Infantil. Conclusión: En conclusión del 100\% de casos y seguimiento en estudio el 96\% se benefició de Aplicación de Evaluación Clínica descrita por Le Metayer incitando sustento al proceso Terapéutico de Atención e Intervención Temprana y un $4 \%$ no tuvo una evolución ventajosa por ser trastornos Neuromotores Permanentes.

Palabras clave: Potencial Motor Innato, Motricidad Bucofacial, Atención Temprana, Intervención Temprana 